On the presence of Apiin in Indian Celery Seeds (Apium graveolens)

The presence of apiin (I) in celery was earlier reported by several authors $\left.{ }^{1}\right)$. But a couple of years back the complete absence of apiin in Indian celery (Karaffs) seeds (Apium graveolens) ${ }^{2}$ ) and the presence of two new anthoxanthin glycosides, graveobioside-A [Luteolin-7-apiosyl glucoside (II)] and graveobioside-B [Chrysoeriol-7-apiosyl glucoside (III)] was reported.

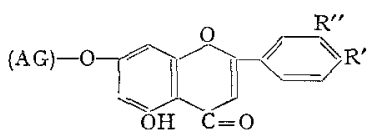

$$
\begin{aligned}
& \text { I } \mathrm{R}^{\prime}=\mathrm{OH} ; \mathrm{R}^{\prime \prime}=\mathrm{H} \\
& \mathrm{II} \mathrm{R}^{\prime}=\mathrm{OH} ; \mathrm{R}^{\prime \prime}=\mathrm{OH} \\
& \mathrm{III} \mathrm{R}^{\prime}=\mathrm{OH} ; \mathrm{R}^{\prime \prime}=\mathrm{OCH}_{3} \\
&(\mathrm{AG})=\text { Apiosyl-glucosyl }
\end{aligned}
$$

In a recent publication ${ }^{3}$ ) on the anthoxanthin glycosides of Apium petroselinum, apiin was assumed to be the only component but on chromatographic examination it was revealed that there are present at least three components ${ }^{4}$, only one of them being apiin. This suggested to the authors to reexamine one of the products of Indian celery (Karaffs) namely graviobioside-B which melted over a range of two degrees $\left(214-16^{\circ}\right)$ in a sulphuric acid bath. When examined on a KOFLER's block under the microscope (which has since become available) the behaviour of the product on melting was not uniform. In fact it had a long range of about ten degrees. It was therefore re-investigated by paper chromatography

Paper chromatography of graveobioside-B using Whatman filter paper No. 1 and No. 3 and the same technique as described earlier ${ }^{4}$ ) led to the conclusion that the graveobioside-B is a mixture of two components which have been identified as apiin (I) by an alongside chromatography with an authentic sample obtained from Apium petroselinum. The identity of the second component was confirmed as chrysoeriol-7 apiosyl glucoside (III) by the identification of its hydrolytic products by using alongside chromatography with authentic samples of chrysoeriol-apiose and glucose.

Further work on the quantitative side is in progress. India

Department of Chemistry Mustim University, Aligarh,

M.O. Faroog, I.P. VARSHNEy and W. RAHMAN

Eingegangen am 21. März 1958

1) Braconnot: Ann. Chim. Phys. 111, 9, 250 (1843). - Schmidt: Pharmazeut. Chem. 2, 2107 (1923). - WEHMER: Pflanzenstoffe 2, 876. - Sannie and Sauvain: Les couleurs cles fleurs et des fruits Anthocyannes et Flavones. Mém. Mus. Nat. Hist. Natur., Paris 1952, 189, - 2) FAROOD GUPTA, KIAMUDDIN, RAHMAN and SesgADRI: J. Sci. Ind. Res. India B 12, $400(1953) .-{ }^{3}$ ) GuPTA and Gadri: J. Sci. Ind. Res. India B 12, $400(1953) .-3)$ GUPTA and
Seshadri: Proc. Indian Acad. Sci. A 35, $242(1952) .-4)$ Faroog, VARSHNEX and Rahman: Naturwiss. 44, 444 (1957).

\section{Enzymatische Phosphorylierung von Myo-Inosit durch ein Enzym aus Hefe}

Es wird allgemein angenommen, daß niedere Phosphorylierungsprodukte des Myo-Inosits als Zwischenprodukte bei der Biosynthese der Phosphoinositide (Phospholipoide, welche Inosit enthalten) fungieren ${ }^{1}$ ). Diese Vorstellung wird auch dadurch unterstützt, daß vor kurzem der Nachweis von freiem Inositmonophosphat in der Leber verschiedener Tiere, im Rinderherz und im Rinderhirn gelungen ist ${ }^{2}$ ). HÜBSCHER und HAWTHORNE berichten in einem kurzen Absatz ihrer Arbeit ${ }^{2}$, ohne dabei experimentelle Details anzugeben, daß sie Beweise fuir die Existenz eines Enzyms in Extrakten aus Rattenleber besitzen, welches die Bildung von Inositmonophosphat aus Adenosintriphosphat (ATP) und Inosit katalysiert. Dies ist unseres Wissens der einzige in der Literatur auffindbare Hinweis auf die enzymatische Synthese von Inositmonophosphat, wenn wir davon absehen, daß diese Substanz natürlich auch beim hydrolytischen Abbau des Phytins durch den in Pflanzen weit verbreiteten Enzymtypus der Phytasen entstehen $m u B^{3}$ ).

Wir beobachteten nun vor kurzem, daß ein zwar einigermaßen konzentriertes, aber von Verunreinigungen nicht freie Präparat einer sog. Hexokinase aus Hefe (,Hexokinase crude, type II from yeast"), das uns die Sigma Chemical Corporation, St. Louis, Missouri, in freundlicher Weise zur Verfügung stellte, imstande ist, die Reaktion

$$
\text { ATP + Myo-Inosit } \rightarrow \text { ADP + Inositmonophosphat }
$$

zu katalysieren. Der Nachweis dieser Wirkung wurde auf zwei Wegen erbracht:
1. Azidimetrische Methode. Pro Mol Phosphat, das von ATP auf Inosit übertragen wird, $m u B$ ein Aquivalent Protonen in Freiheit gesetzt werden. Die Säurebildung läßt sich durch direkte Titration mit Natronlauge verfolgen ${ }^{4}$, ${ }^{5}$ ). Wir folgten in der Ausführung der von McDoNaID ${ }^{6}$ ) angegebenen Vorschrift.

2. Direkter Nachweis der Bildung von Inositmonophosphat. Dieser konnte dadurch erbracht werden, daß man das entstandene Inositmonophosphat vom freien Inosit mit Hilfe eines basischen Ionenaustauschers trennte, das Inositphosphat nach der Elution zu Inosit verseifte und diesen papierchromatographisch nachwies. 2,1 $\mathrm{mg}$ Inosit, $32,5 \mathrm{mg}$ ATP und $0,2 \mathrm{mg}$ $\mathrm{MgCl}_{2}$ wurden in 0,2 molarem Tris-HCl-Puffer von $\mathrm{p}_{\mathrm{H}} 7,5$ gelöst und 1 Std lang mit der Enzymlösung inkubiert (Gesamtvolumen $3 \mathrm{ml}$ ). Die Reaktion wurde durch Zugabe von $2 \mathrm{ml}$ $10 \%$ iger Trichloressigsäure gestoppt und die Nucleotide durch Adsorption an aktiviertem Norit A entfernt ${ }^{7}$ ). Die Reaktionsmischung wurde mit Dowex $1 \times 10$ (200 bis 400 mesh) behandelt, der Austauscher mit Wasser inosit-frei gewaschen, das Inositphosphat mit Ameisensäure eluiert und schlieBlich durch 20stündige Hydrolyse in $5,8 \mathrm{n} \mathrm{HCl}$ bei $110^{\circ}$ unter Drack in Inosjt überführt ${ }^{2}$ ). Der Inosit konnte dann papierchromatographisch [System: Ameisensäure-Aceton-Wasser; aufsteigend, nach Hurchison u. Mitarb. $\left.\left.{ }^{8}\right)\right]$ nachgewiesen werden; die Inositflecken wurden mit alkalischer Silbernitratlösung sichtbar gemacht ${ }^{9}$ ).

Arbeiten zur weiteren Charakterisierung der Enzymaktivität und insbesondere zur Entscheidung der Frage, ob es sich hier um eine Nebenaktivität der Hexokinase oder um einen eigenen Enzymtypus, eine Inositkinase (rationeller Name: ATP-Inosit-Phosphoryltransferase), handelt, sind zur Zeit im Gange.

Wien, I. Chemisches Institut dev Universität

O. Hoffmann-OstenhoF, C. Jungwirth und I.B. DAWid

Eingegangen am 29. März 1958

1) KenNedy, E.P., u. S.B. Weiss: J. Biol. Chern. 222, 193 (1956). - 2) HỨBscher, G., u. J.N. HAWTHORNE: Biochemic. J. 67, 523 (1957). - 3) Vgl. z.B. Fleury, P., u. J. Courtois: Helv. chim. Acta 29, 1297 (1946). - 4) Colowick, S.P., u. H.M. Kalckar: J. Biol. Chem. 137, $789(1941) ; 148,117(1943)$. - 5) KUnitz M., U. M.R. McDonald: J. Gen, Physiol. 29, 393 (1946). - 6) MCDoNALD, M.R.: In: Methods in Enzymology, Bd. I, S. 269 . New York: Academic Press 1955. - 7) Crane, R. K., u. F. LipManN: J. Biol. Chem. 201, 235 (1953). - - $^{2}$ Hutchison, W. C., G.W. Crosbie, C.B. Mendes, W.M. McIndoe, M. Childs u. J.N. DAvidson: Biochim. et Biophys. Acta 21, 44 (1956). - 9) TrEvelyaN, W.E., D.P. Procter u. J.S. HAarrison: Nature [London] 166, 444 (1950).

\section{Über die Anthocyanverteilung in bunten Laubblättern}

In früheren Untersuchungen haben wir ausführlich den Zusammenhang dargestellt, der zwischen der Ausbildung von Silberflecken auf der Oberseite von Blättern und dem Fehlen von Anthocyan (Cyanidinglycoside) auf der Blattunterseite besteht ${ }^{\mathbf{1}}$ ), ${ }^{2}$ ). Aber nicht nur bei Begonien, sondern auch bei Melastomataceen, Gesneriaceen und Piperaceen konnten wir neuerdings eine derartige Beziehung konstatieren. Sie tritt sowohl unabhängig von der spezifischen anatomischen Struktur, die die Silberfleckenzeichnung hervorruft, als auch unabhängig von der Art des Anthocyans auf; denn bei den untersuchten Melastomataceen finden sich als Aglucon des Anthocyans vor allem Malvidin und Delphinidin an Stelle des Cyanidins, das bei Begonien ausschlieBlich vorkommt. Im Unterschied zu Begonienarten ist bei einigen Vertretern der oben genannten Familien zwar der Gehalt an Anthocyan unter den Silberflecken herabgesetzt, gleichzeitig sind aber Flavonole in wesentlich erhöhter Menge vorhanden, was wir auf papierchromatographischem Wege genau nachweisen konnten. Es liegt hier eine lklare alternative Ausbildung der beiden Flavanderivate, dem Anthocyan einerseits und dem Flavonol andererseits, vor, worauf an anderer Stelle noch ausführlich eingegangen werden soll.

Wir erklärten die enge Beziehung zwischen Silberflecken und Anthocyanverteilung durch eine Schattenwirkung der Silberflecken ${ }^{1}$, indem aneinanderschließende Interzellularen, unter der Epidermis ${ }^{3}$ ) oder in einer Schicht von Palisadenhomologen, das Licht reflektieren ${ }^{4}$ ), so daB die Menge des durchgelassenen Lichtes in den unteren Blattschichten (Mesophyll und Epidermis) wesentlich vermindert ist. Die Verminderung der Lichtintensität hat aber im allgemeinen eine geringere Anthocyansynthese zur Folge. 